\title{
Electron collisions with excited molecules in low temperature plasmas
}

\author{
Roberto Celiberto ${ }^{1,2, a}$ and Vincenzo Laporta ${ }^{2,3}$ \\ ${ }^{1}$ Dipartimento di Ingegneria Civile, Ambientale, del Territorio, Edile e di Chimica, Politecnico di Bari, \\ Italy \\ ${ }^{2}$ Istituto di Metodologie Inorganiche e Plasmi, CNR, Bari, Italy \\ ${ }^{3}$ Department of Physics and Astronomy, University College London, London WC1E 6BT, UK
}

\begin{abstract}
State-to-state vibrationally resolved cross sections for electron-impact processes involving vibrationally excited molecules are reviewed, with particular emphasis on atmospheric and fusion plasma applications.
\end{abstract}

\section{Introduction}

In non-equilibrium low-temperature molecular plasmas the densities of active species (molecules, atoms, ions...) can strongly deviate from the Boltzmann distribution. Each internal rovibronic state of the molecules acts in the plasma as a single species, with its own processes, so that the number of active molecules largely exceed the number of true chemical species. A theoretical model of a molecular plasma to determine the particle densities, requires then, as input data, huge sets of cross sections for all those collisional processes which, in principle, can involve all the internal molecular states accessible in the system (the so-called state-to-state approach [1]).

A role of central relevance is played by the electron collisions with vibrationally excited molecules, which, through inelastic and superelastic exchanges of energy, can deeply affect both the vibrational population and the electron energy distribution function [2].

In this contribution we briefly review electron-impact cross section data sets, obtained by theoretical calculations, for processes involving vibrationally excited molecules, with particular emphasis on aerospace and nuclear fusion applications $[3,4]$.

The paper is organized in two general parts: in Sect. 2 we will focus on electron-molecule collision processes involving the chemical components of atmospheric plasmas while, in Sect. 3, we will deal with molecular species relevant for fusion plasmas.

\section{Atmospheric gases}

In re-entry conditions, that is when a space vehicle returns back to the Earth, the thermal shield interacts with the terrestrial atmosphere whose main components are diatomic nitrogen and oxygen and, in small

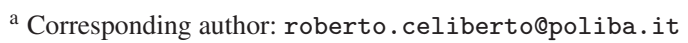

This is an Open Access article distributed under the terms of the Creative Commons Attribution License 4.0, which permits unrestricted use, distribution, and reproduction in any medium, provided the original work is properly cited. 


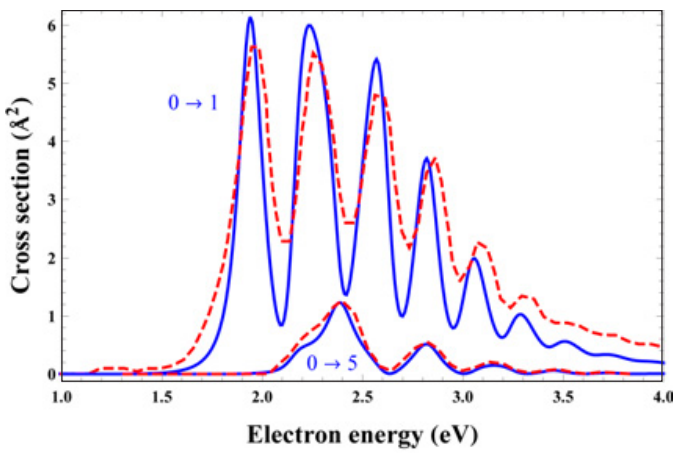

Figure 1. Electron- $\mathrm{N}_{2}$ theoretical-experimental cross section comparison for the $v_{i}=0 \rightarrow 1,5$ transitions. Full lines: theoretical calculations [5]; dashed lines: experimental results [6].

fraction, also $\mathrm{NO}, \mathrm{CO}$ and $\mathrm{CO}_{2}$ molecules. We will review in Sect. 2.1 electron-impact collision cross sections for vibrational excitations of nitrogen, oxygen and nitric oxide, while cross sections for carbon monoxide and dioxide, which are also the most abundant species of the Mars and Venus atmospheres, of interest in planetary explorations, will be discussed in Sect. 2.2.

\subsection{Electron collisions with $\mathrm{N}_{2}, \mathrm{O}_{2}$ and $\mathrm{NO}$ molecules}

Resonant vibrational excitation (RVE) cross sections have been calculated for electron-nitrogen scattering

$$
\mathrm{N}_{2}\left(\mathrm{X}^{1} \Sigma_{\mathrm{g}}^{+}, v_{i}=0-67\right)+e \rightarrow \mathrm{N}_{2}^{-}\left(\mathrm{X}^{2} \Pi_{\mathrm{g}}\right) \rightarrow \mathrm{N}_{2}\left(\mathrm{X}^{1} \Sigma_{\mathrm{g}}^{+}, v_{f}=0-67\right)+e,
$$

which proceeds through the resonant state $\mathrm{N}_{2}^{-}\left(\mathrm{X}^{2} \Pi_{\mathrm{g}}\right)$. RVE cross sections for this process have been calculated for all the possible transitions among the 68 vibrational levels supported by the electronic ground state. Potential curves for $\mathrm{N}_{2}$ and $\mathrm{N}_{2}^{-}$were expressed as Morse functions while the width was obtained semi-empirically [5]. Good agreement is found with the experimental data of Ref. [6] as shown in Fig. 1, where the cross sections for the transitions $0 \rightarrow 1$ and $0 \rightarrow 5$ are represented as a function of the incident electron energy.

Figure 2 shows the cross sections for the elastic and inelastic $20 \rightarrow v_{f}$ transitions. The rapid oscillations are generated by the vibrational wave functions of the resonant states $\mathrm{N}_{2}^{-}\left(\mathrm{X}^{2} \Pi_{\mathrm{g}}\right)$ and disappear above the dissociation energy of the $\mathrm{N}_{2}^{-}$potential curve where its continuum starts.

RVE cross sections by electron-impact have also been calculated for vibrationally excited oxygen molecules, according to the process [7]:

$$
e+\mathrm{O}_{2}\left(\mathrm{X}^{3} \Sigma_{\mathrm{g}}^{-}, v_{i}=0-41\right) \rightarrow \mathrm{O}_{2}^{-}\left({ }^{2} \Pi_{\mathrm{g}},{ }^{2} \Pi_{\mathrm{u}},{ }^{4} \Sigma_{\mathrm{u}}^{-},{ }^{2} \Sigma_{\mathrm{u}}^{-}\right) \rightarrow e+\mathrm{O}_{2}\left(\mathrm{X}^{3} \Sigma_{\mathrm{g}}^{-}, v_{f}=0-41\right)
$$

The reaction follows the same mechanism as in process (1) for $\mathrm{N}_{2}$, but in this case four resonant states contribute to the vibrational excitations. Potential curves for these states and for the ground state of the target molecule, as well as the four widths, were obtained as described in Ref. [7]. The cross sections were calculated for all the possible transitions $\left(v_{i}, v_{f} \leq 41\right)$. Comparison of calculated and experimental cross sections, for the transitions $0 \rightarrow v_{f}=1,2,3$ and 4, is shown in Fig. 3. Good agreement is observed with the measurements of Noble et al [8] for the $0 \rightarrow 1$ and $0 \rightarrow 2$ transitions, while the corresponding theoretical results of the same authors show some discrepancy, probably due to the fact that only the ${ }^{4} \Sigma_{\mathrm{u}}^{-}$resonant state is considered in their calculations. A larger discrepancy is instead observed with the experiments for the $0 \rightarrow 3$ and $0 \rightarrow 4$ transitions. This difference can be probably ascribed to the fact 

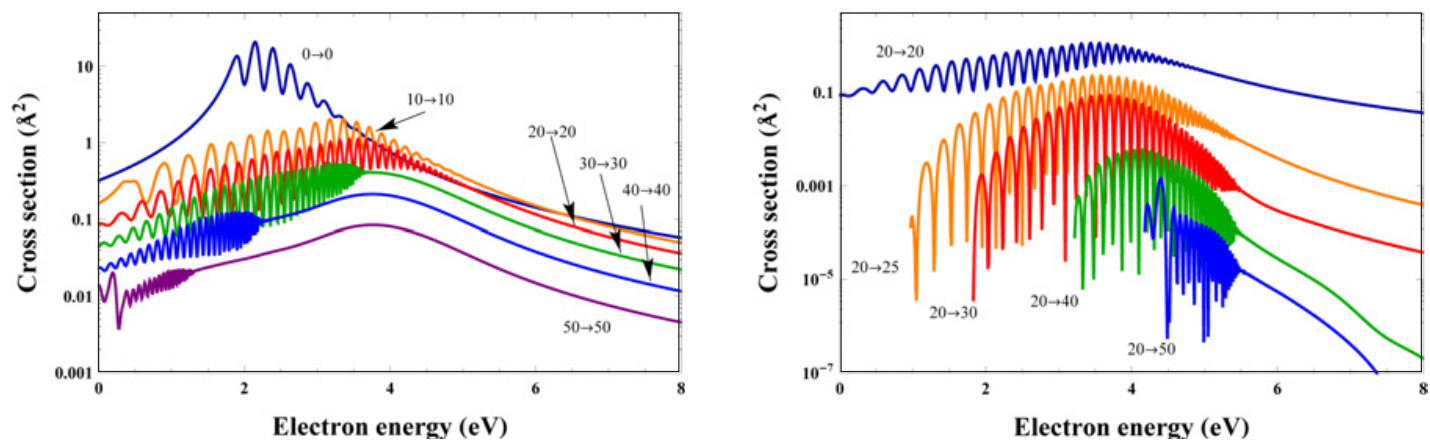

Figure 2. $\mathrm{N}_{2}$ vibrational-excitation cross sections for the elastic $v_{i}=v_{f}$ (left panel) and inelastic $v_{i}=20 \rightarrow v_{f}$ (right panel) transitions [5].
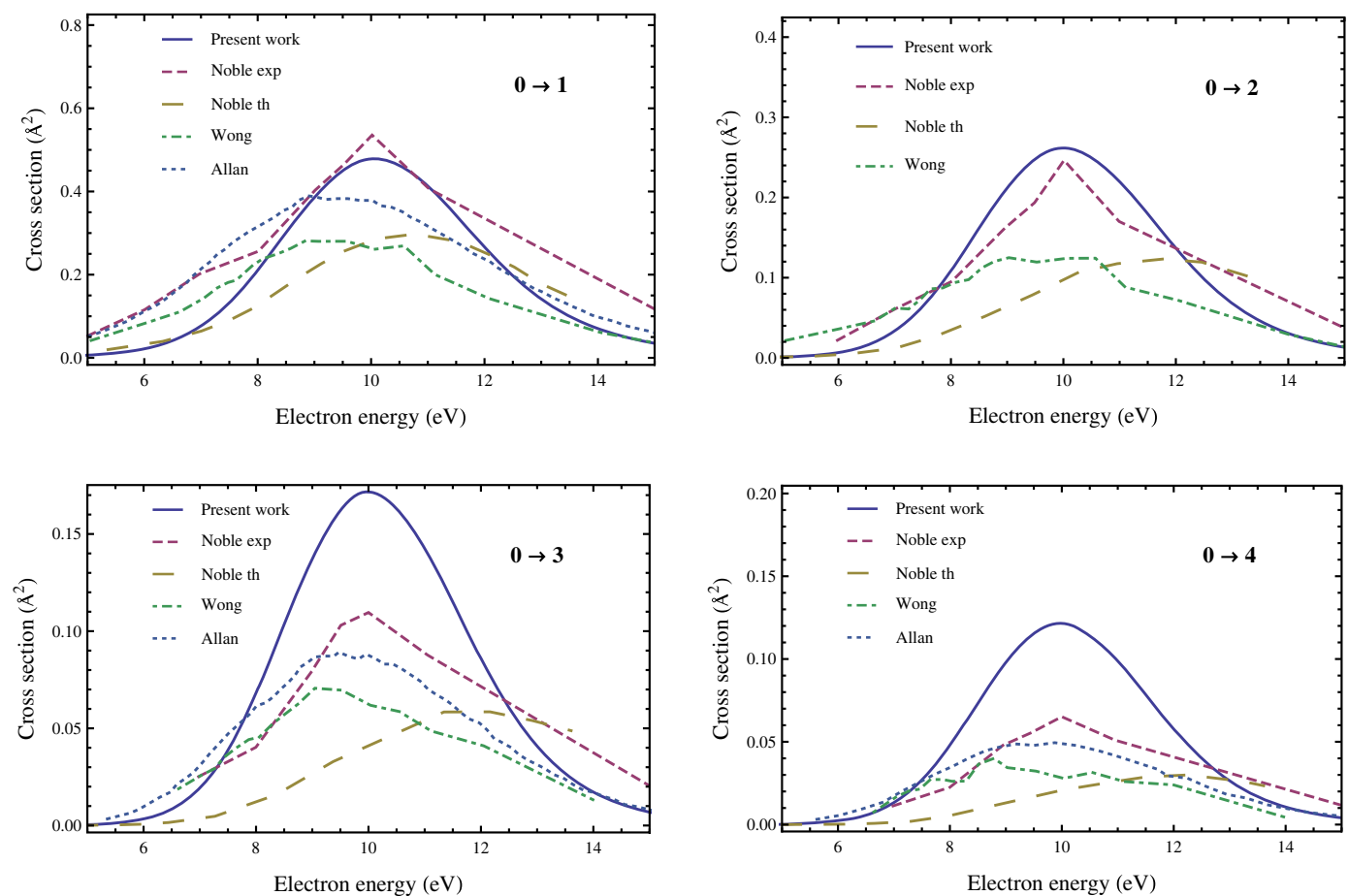

Figure 3. Electron- $\mathrm{O}_{2}$ vibrational-excitation cross sections. Comparison of the calculated cross sections of Laporta et al. [7] (solid lines) with experimental (dashed lines) and theoretical (long-dashed lines) results of Noble et al. [8], Wong et al.'s measurements (dotted-dashed lines) [8] and Allan's experiments (dotted lines) [9].

that the integrated cross section of Fig. 3 were obtained by the corresponding differential cross sections by assuming, for the outgoing electron, a $p$-wave nature $(l=1)$, while a mixed contribution of $l=1$ and $l=2$ partial waves would have been more appropriate $[7,9]$.

Figure 4 shows the cross sections for process (2) for some elastic and inelastic transitions. The different behavior at low and high electron energy, is due to the dominant contribution of different resonant states. In particular, the strong oscillations below $4 \mathrm{eV}$ are mainly caused by $\mathrm{O}_{2}\left({ }^{2} \Pi_{\mathrm{g}}\right)$ while the broad structure observed around $10 \mathrm{eV}$ for the $0 \rightarrow v_{f}$ transitions is attributed to the dominance of 

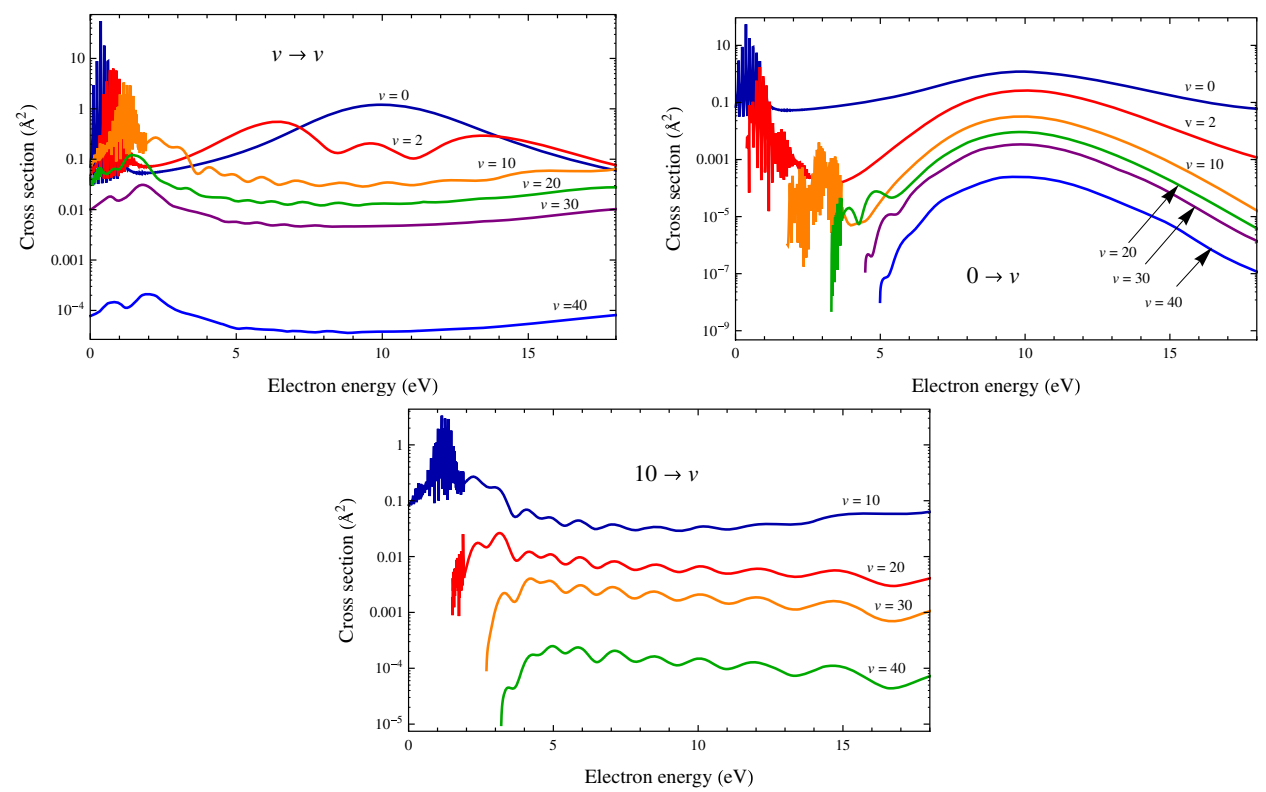

Figure 4. Electron- $\mathrm{O}_{2}$ RVE cross sections for the elastic and inelastic transitions as shown in the plots [7].

$\mathrm{O}_{2}\left({ }^{4} \Sigma_{\mathrm{u}}^{-}\right)$state. The oscillations for the $10 \rightarrow v$ above $\sim 2 \mathrm{eV}$, in the third panel, can be attributed to the overlap of the vibrational wave function for the level 10 of the neutral molecule with the continuum spectrum of the $\mathrm{O}_{2}^{-}$ion [7].

Electron-impact cross sections have also been calculated for vibrational excitations of nitric oxide according to the process

$$
e+\mathrm{NO}\left(\mathrm{X}^{2} \Pi, v_{i}=0-54\right) \rightarrow \mathrm{NO}^{-}\left({ }^{3} \Sigma^{-},{ }^{1} \Delta,{ }^{1} \Sigma^{+}\right) \rightarrow e+\mathrm{NO}\left(\mathrm{X}^{2} \Pi, v_{f}=0-54\right) .
$$

In this case three resonant states contribute to the RVE process. As for nitrogen the potential curves for the $\mathrm{NO}$ ground state and the $\mathrm{NO}^{-}$resonant states were expressed as a Morse functions, while the widths were set empirically [5]. Comparison with the experiments is shown in Fig. 5.

Although the peak positions are reproduced, some discrepancy is observed in their intensities. However, the same theoretical cross sections are in good agreement with those of Trevisan et al. [11] as shown in Fig. 6. This implies that the disagreement is between theory and experiments. Example of elastic and inelastic cross sections are shown in Fig. 7.

\subsection{Electron collisions with $\mathrm{CO}$ and $\mathrm{CO}_{2}$ molecules}

Cross sections for electron-impact vibrational excitation involving the $\mathrm{CO}$ molecule have been computed by ab initio calculations. The RVE process can be written as:

$$
e+\mathrm{CO}\left(\mathrm{X}^{1} \Sigma^{+}, v_{i}=0-80\right) \rightarrow \mathrm{CO}^{-}\left({ }^{2} \Pi\right) \rightarrow e+\mathrm{CO}\left(\mathrm{X}^{1} \Sigma^{+}, v_{f}=0-80\right) .
$$

Potential curve for the $\mathrm{CO}$ ground electronic state has been obtained by structure calculations using the quantum chemistry code MOLPRO [12], while the resonant state $\mathrm{CO}^{-}\left({ }^{2} \mathrm{P}\right)$ potential energies and the width were calculated by the $R$-matrix method [13]. The cross sections were found to be in good agreement with previous theoretical calculations [14] and with the experiments [15] as shown in Fig. 8. Some discrepancy, however, is observed with the experiments for the $0 \rightarrow 10$ excitation. 

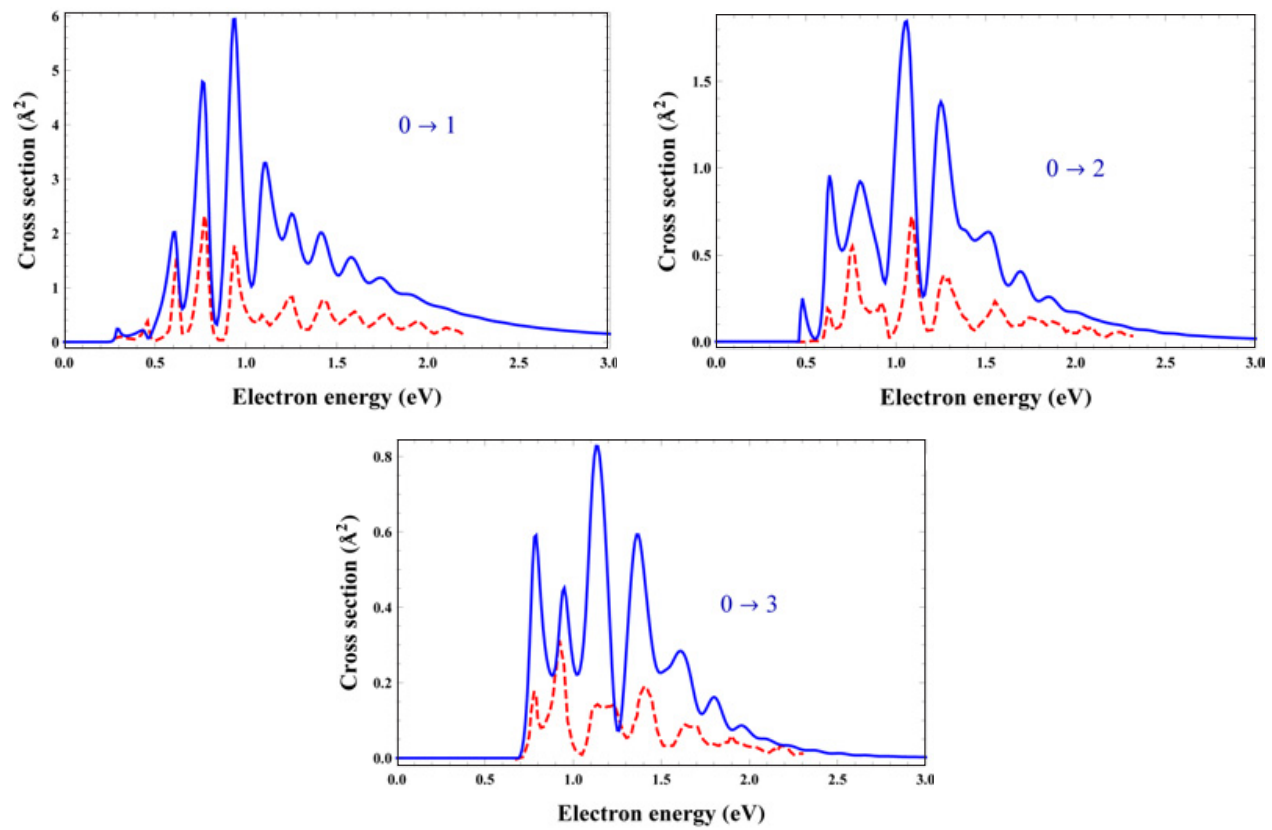

Figure 5. Electron-NO comparison of the calculated RVE cross sections [5] (full lines) with the measurements [10] (dashed lines) for the inelastic vibrational transitions $0 \rightarrow 1,2,3$.
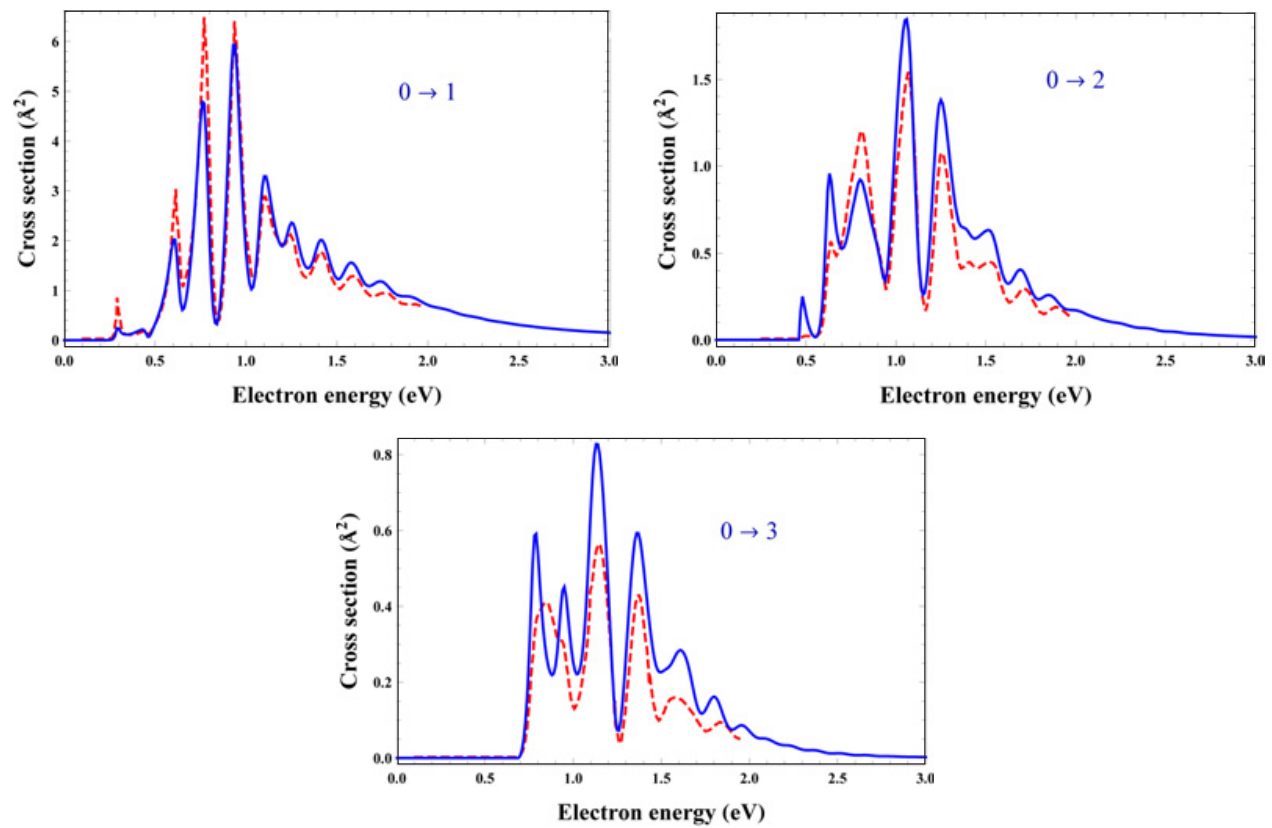

Figure 6. Electron-NO comparison of the theoretical RVE cross sections of Laporta et al. [5] (full lines) with the calculations of Trevisan et al. [11] (dashed lines) for the inelastic vibrational transitions $0 \rightarrow 1,2,3$. 

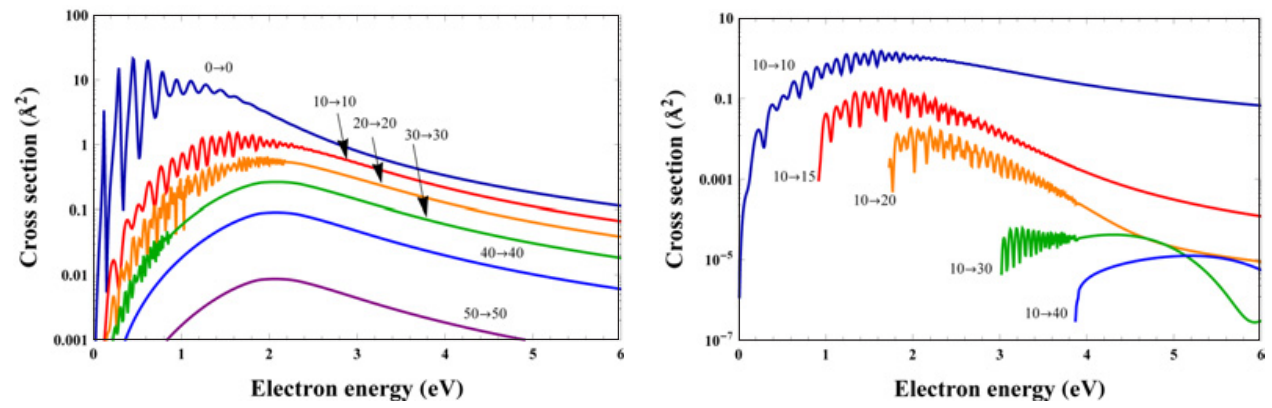

Figure 7. Electron-NO RVE cross sections for elastic and inelastic transitions as shown in the panels [5].
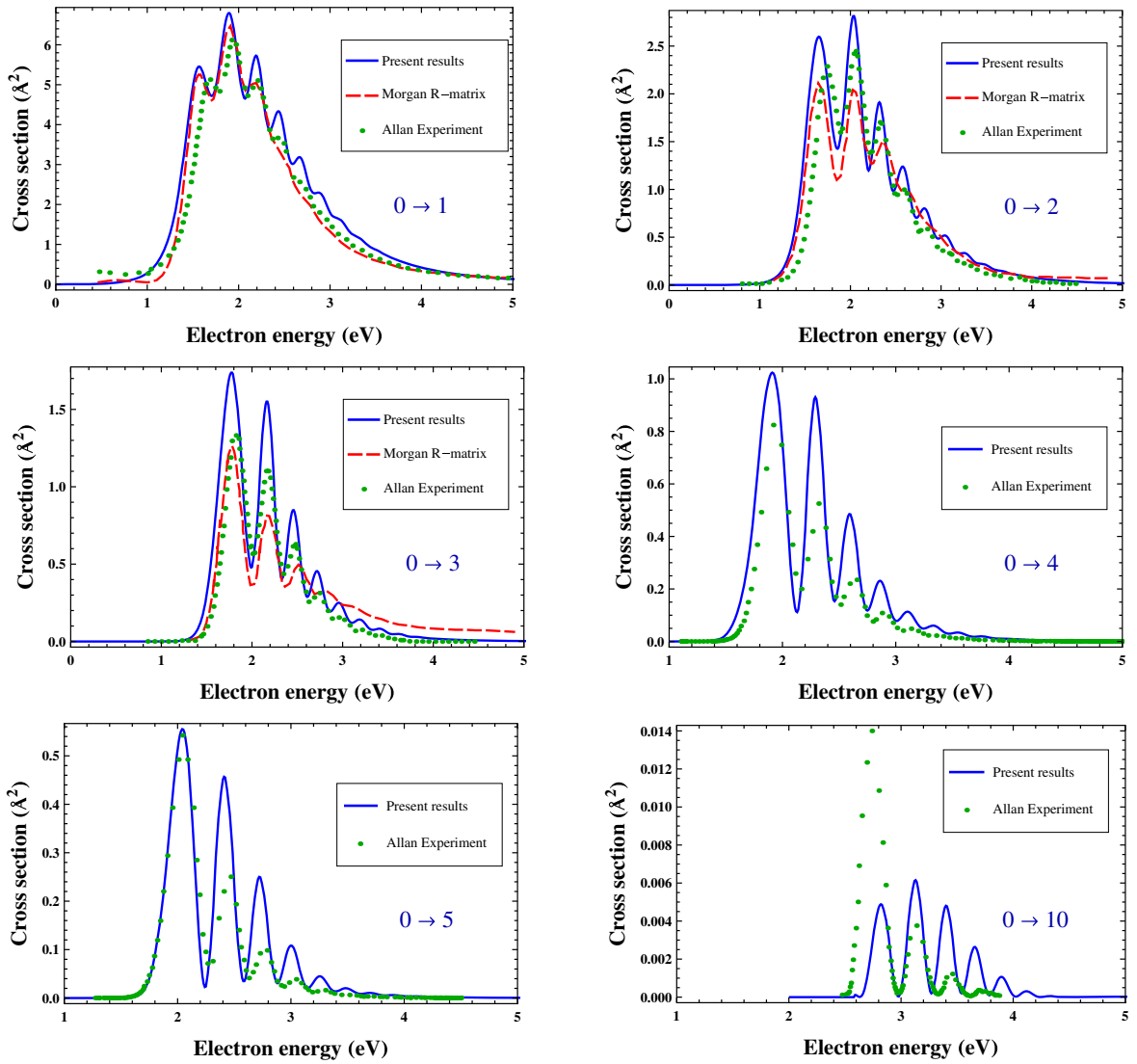

Figure 8. Electron-CO resonant cross sections from Ref. [13] compared with the experimental results of Ref. [15] and the theoretical $R$-matrix calculation of Ref. [14].

Elastic and inelastic cross sections for some RVE transitions are shown in Fig. 9.

$\mathrm{CO}_{2}$ is a triatomic linear molecule characterized by three vibrational normal modes, so that a theoretical cross section calculation should then be based on a multidimensional treatment [16]. However, according to the experimental observations of Čadez et al. [17], the stretching mode can be approximately separated from the asymmetric and bending modes, so that a one-dimensional model 

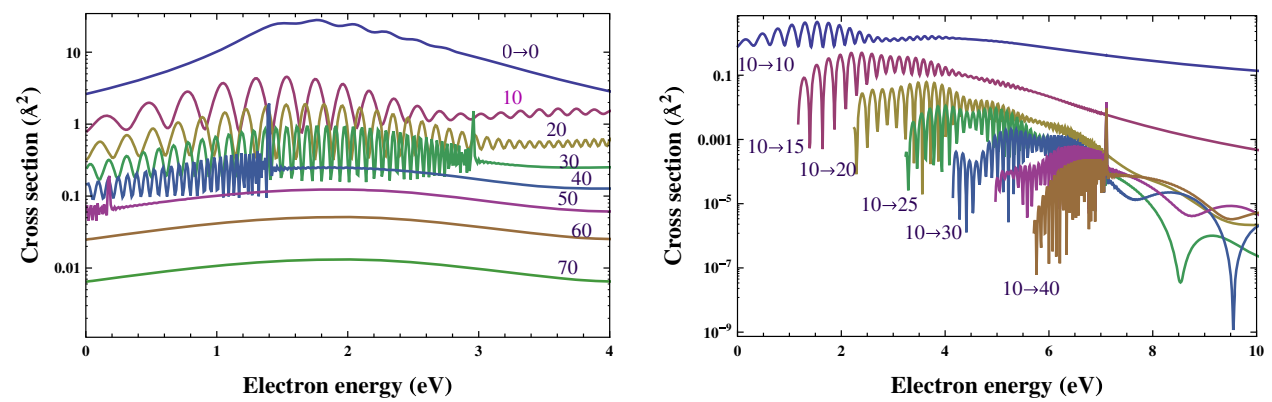

Figure 9. Electron-CO RVE cross sections for elastic and inelastic transitions as shown in the panels [13].
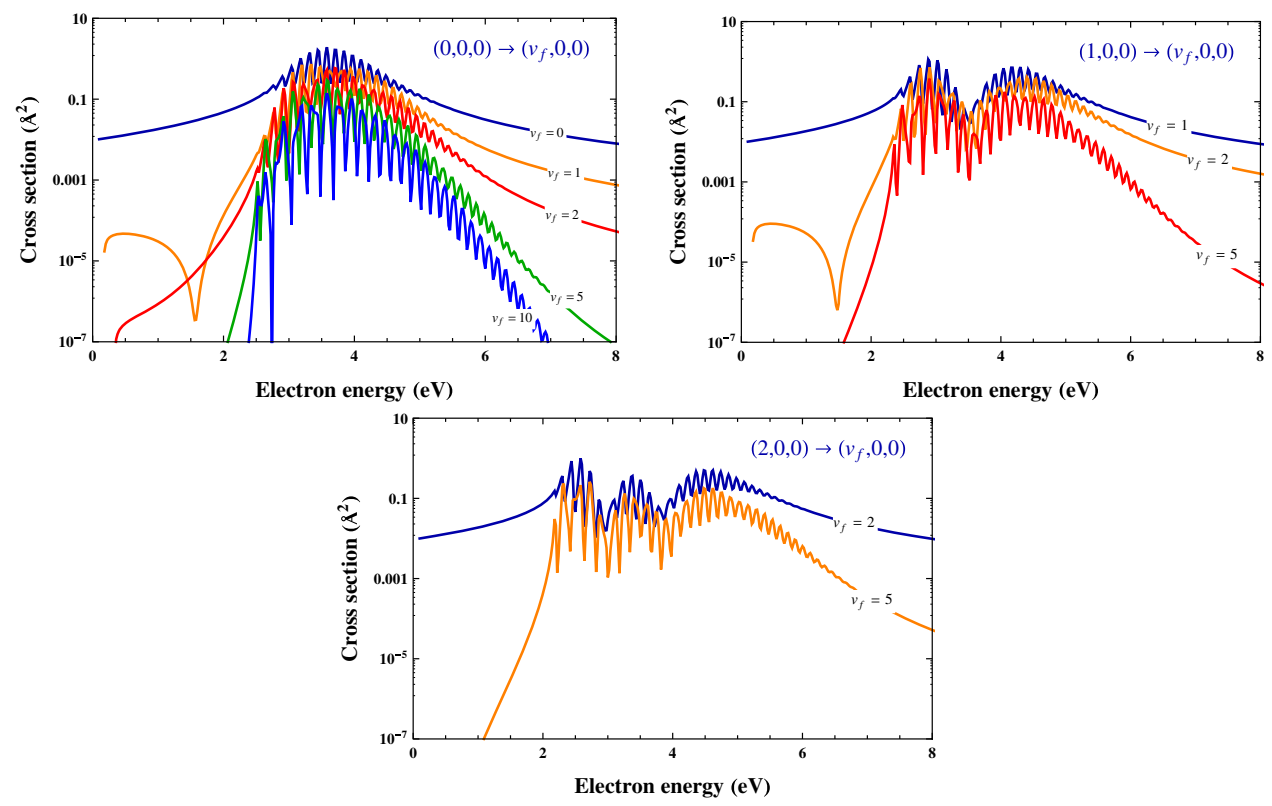

Figure 10. Electron- $\mathrm{CO}_{2}$ RVE cross sections for the transitions $0 \rightarrow v_{f}=0,1,2,5,10,1 \rightarrow v_{f}=1,2,5$ and $2 \rightarrow v_{f}=2,5$, as shown in the three panel respectively [18].

can be used for cross sections calculations. Assuming thus the vibrational decoupling, we have obtained preliminary results for the process

$$
\mathrm{CO}_{2}\left(\mathrm{X}^{1} \Sigma_{\mathrm{g}}^{+}, v_{i}, 0,0\right)+e \rightarrow \mathrm{CO}_{2}^{-}\left({ }^{2} \Pi_{\mathrm{u}}\right) \rightarrow \mathrm{CO}_{2}\left(\mathrm{X}^{1} \Sigma_{\mathrm{g}}^{+}, v_{f}, 0,0\right)+e,
$$

involving only the symmetric stretching excitation $v_{i} \rightarrow v_{f}$. Potential curves for $\mathrm{CO}_{2}$ and $\mathrm{CO}_{2}^{-}$and widths were obtained by ab initio calculations [18]. In Fig. 10 the cross sections for the transitions $v_{i}=0 \rightarrow v_{f}=0,1,2,5,10, v_{i}=1 \rightarrow v_{f}=1,2,5$ and $v_{i}=2 \rightarrow v_{f}=2,5$ are shown.

\section{Fusion plasmas}

The relatively low temperatures of the edge and divertor regions of fusion reactors, characterized by the presence of molecular hydrogen, allow the formation of new molecular species generated by plasma-wall interactions. In current experimental reactors, beryllium and carbon-graphite are utilized as 

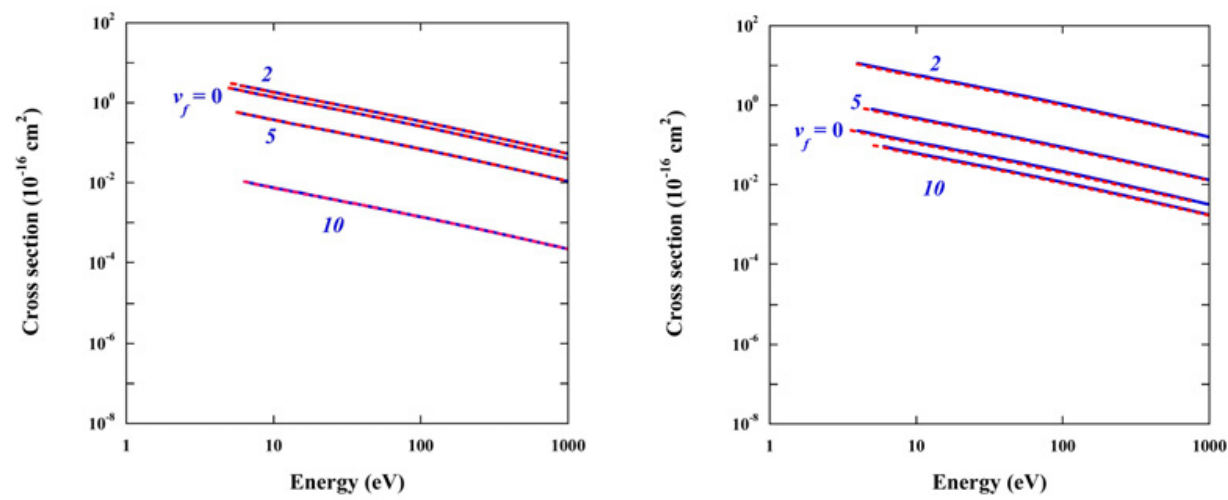

Figure 11. Electron- $\mathrm{BeH}^{+}$cross sections (full-blue lines) for the processes $\mathrm{X}^{1} \Sigma^{+}\left(v_{i}=0\right) \rightarrow \mathrm{A}^{1} \Sigma^{+}\left(v_{f}\right)$ (left panel) and $\mathrm{X}^{1} \Sigma^{+}\left(v_{i}=5\right) \rightarrow \mathrm{A}^{1} \Sigma^{+}\left(v_{f}\right)$ (right panel), for $v_{f}=0,2,5,10$ [19]. The dashed-red lines represent the scaled cross sections calculated by Eq. (8).

plasma-facing materials for the main tokamak chamber and divertor plates, so that plasma-surface chemical reactions yield hydrocarbons and beryllium hydrates. The modeling of the edge and divertor plasmas requires therefore molecular and scattering data for these compounds.

We will review the recent electron-impact theoretical cross sections for vibro-electronic excitation of $\mathrm{BeH}^{+}$and $\mathrm{BeH}$ molecules for the following processes [19-21],

$$
\begin{aligned}
& \mathrm{BeH}^{+}\left(\mathrm{X}^{1} \Sigma^{+}, v_{i}\right)+e \rightarrow \mathrm{BeH}^{+}\left(\mathrm{A}^{1} \Sigma^{+}, \mathrm{B}^{1} \Pi, v_{f}\right)+e, \\
& \operatorname{BeH}\left(\mathrm{X}^{2} \Sigma^{+}, v_{i}\right)+e \rightarrow \operatorname{BeH}\left(\mathrm{A}^{2} \Pi, v_{f}\right)+e .
\end{aligned}
$$

The cross section for process (6) has been calculated using the Coulomb-Born approximation [19], which requires the dipole transition moments and potential curves for the electronic states involved in the transitions. These quantities were provided in Ref. [22]. The calculated cross sections for the $\mathrm{X}^{1} \Sigma^{+} \rightarrow \mathrm{A}^{1} \Sigma^{+}$and $\mathrm{X}^{1} \Sigma^{+} \rightarrow \mathrm{B}{ }^{1} \Pi$ electronic transitions and for some selected $v_{i}, v_{f}$ vibrational levels are shown in Fig. 11 (full-blue lines).

In the same figure are shown the cross sections (dashed-red lines) calculated by the following analytical formula

$$
\sigma_{v_{i}, v_{f}}^{\mathrm{X}, \mathrm{A}}\left(x \cdot \Delta \mathrm{E}_{v_{i}, v_{f}}\right)=\frac{\Delta \mathrm{E}_{0,0}}{\Delta \mathrm{E}_{v_{i}, v_{f}}}\left(\frac{\mathrm{M}_{v_{i}, v_{f}}}{\mathrm{M}_{0,0}}\right)^{2} \cdot \sigma_{0,0}^{\mathrm{X}, \mathrm{A}}\left(x \cdot \Delta \mathrm{E}_{0,0}\right)
$$

where the transition energy $\Delta \mathrm{E}_{v_{i}, v_{f}}$ and the dipole transition moment matrix elements, $\mathbf{M}_{v_{i}, v_{f}}$, are provided in Ref. [19], $x$, the reduced energy, is defined by $x=\epsilon_{i} / \Delta \mathrm{E}_{v_{i}, v_{f}}$ where $\epsilon_{i}$ is the incident electron energy. Eq. (8) permits a rapid and accurate calculation of the $\mathrm{X}\left(v_{i}\right) \rightarrow \mathrm{A}\left(v_{f}\right)$ cross sections by scaling the corresponding cross section, $\sigma_{0,0}^{\mathrm{X}, \mathrm{A}}$, for the $0 \rightarrow 0$ transition.

Cross sections for the $\mathrm{X}^{1} \Sigma^{+}\left(v_{i}=0\right) \rightarrow \mathrm{B}^{1} \Pi\left(v_{f}\right)$ are shown in Fig. 12.

Electron-impact cross sections have also been obtained for process (7). The calculations have been performed at low energies for the transition $\mathrm{X}^{2} \Sigma^{+}\left(v_{i}=0\right) \rightarrow \mathrm{A}^{2} \Pi\left(v_{f}=0\right)$, by using the $R$-matrix method, and were then extended to high energies and to all the vibrational transitions by using a threshold modified version of the Mott and Massey approximation (TMMM approximation) [21]. The use of the two methods is justified by the fact that the $R$-matrix method gives accurate cross sections close to the energy threshold of the process, while the Mott and Massey formulation is appropriate at high energies, typical of fusion plasmas. Moreover, the $R$-matrix calculations can be used to validate the TMMM approximation. $R$-matrix and TMMM results are successfully compared in Fig. 13. 

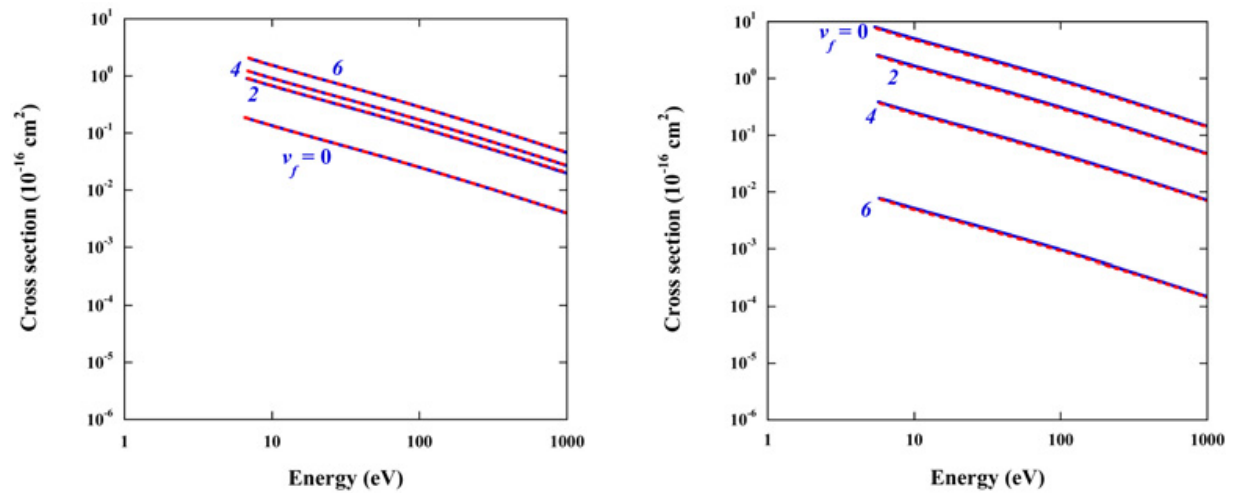

Figure 12. Same as Fig. 11 for the processes $\mathrm{X}^{1} \Sigma^{+}\left(v_{i}=0\right) \rightarrow \mathrm{B}{ }^{1} \Pi\left(v_{f}\right)$ (left panel) and $\mathrm{X}^{1} \Sigma^{+}\left(v_{i}=5\right) \rightarrow$ $\mathrm{B}{ }^{1} \Pi\left(v_{f}\right)$ (right panel) for $v_{f}=0,2,4,6$.

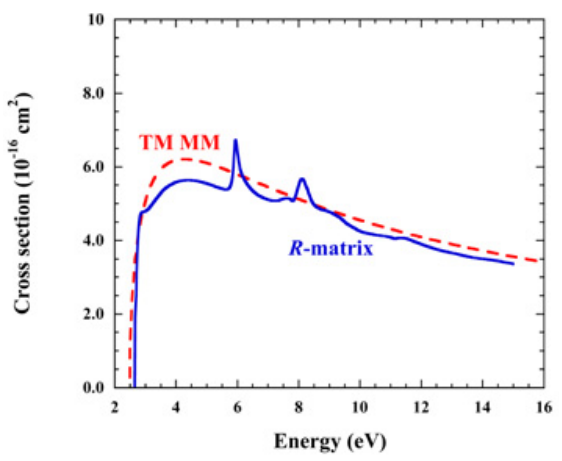

Figure 13. Comparison of the electron-BeH cross sections calculated by using both the $R$-matrix method (full-blue line) and the TMMM approximation (dashed-red line) for the process $\mathrm{X}^{2} \Sigma^{+}\left(v_{i}=0\right) \rightarrow \mathrm{A}^{2} \Pi\left(v_{f}=0\right)$.

We should stress here the fact that the TMMM approximation includes the vibrational motion [21] while the $R$-matrix cross sections are calculated in the usual fixed nuclei approximation at the equilibrium distance of the $\mathrm{Be}-\mathrm{H}$ bond. However, in the $R$-matrix method, we may assume that the vibrational motion is confined in the multiplicative Franck-Condon factor for the $0 \rightarrow 0$ transition, which however has been found to be very close to the unity [21], so that the comparison in Fig. 13 can be considered consistent.

The TMMM approximation has been extended to the calculation of state-to-state cross sections for a range of energy from the threshold to $1000 \mathrm{eV}$. Potential curves and transition dipole moments were taken from Ref. [23]. An example is shown in Fig. 14 for the transitions $\mathrm{X}^{2} \Sigma^{+}\left(v_{i}\right) \rightarrow \mathrm{A}^{2} \Pi\left(v_{f}\right)$ (fullblue lines). Again, in this figure are also shown the scaled cross sections by Eq. (8) (red-dashed lines) with $\Delta \mathrm{E}_{v_{i}, v_{f}}$ and $\mathrm{M}_{v_{i}, v_{f}}$ provided in Ref. [21].

One of the most important process in low temperature fusion plasma, is resonant vibrational excitations involving vibrationally excited $\mathrm{H}_{2}$ molecules. Cross sections for this processes were calculated since the early studies of resonant collisions for the e- $\mathrm{H}_{2}$ scattering occurring through the resonant ground electronic state $\mathrm{H}_{2}^{-}\left(\mathrm{X}^{2} \Sigma_{u}^{+}\right)$[24]. More recently [25], vibrational excitation cross sections were calculated for a resonant collision involving a Rydberg-excited ${ }^{2} \Sigma_{\mathrm{g}}^{+}$resonant state, 

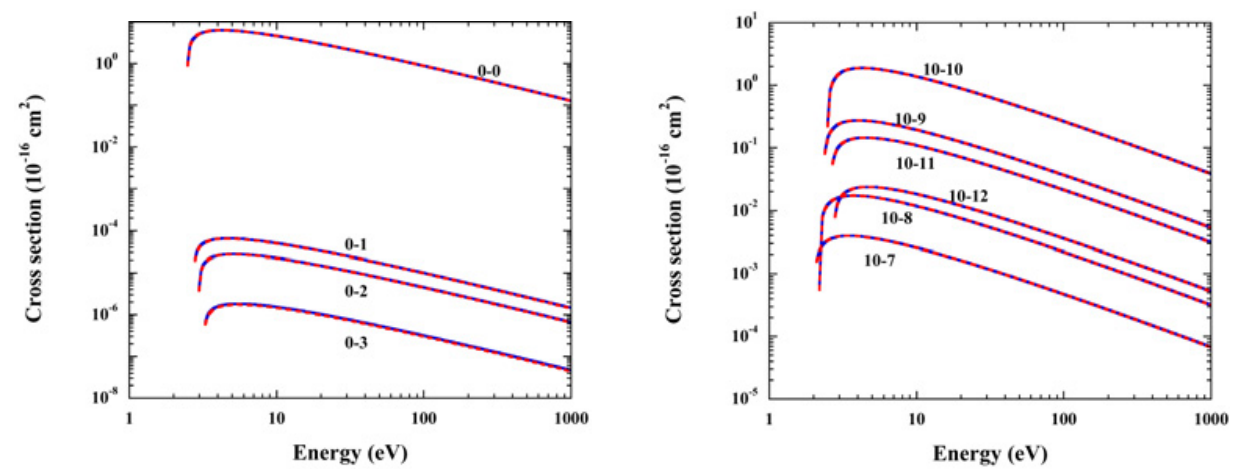

Figure 14. Full-blue lines: TMMM electron-BeH cross sections for the process $\mathrm{X}^{2} \Sigma^{+}\left(v_{i}\right) \rightarrow \mathrm{A}^{2} \Pi\left(v_{f}\right)$ with $v_{i}=0$ (left panel) and $v_{i}=10$ (right panel) and for some selected value for $v_{f}$ [21]. Red-dashed lines: scaled cross sections by Eq. (8).
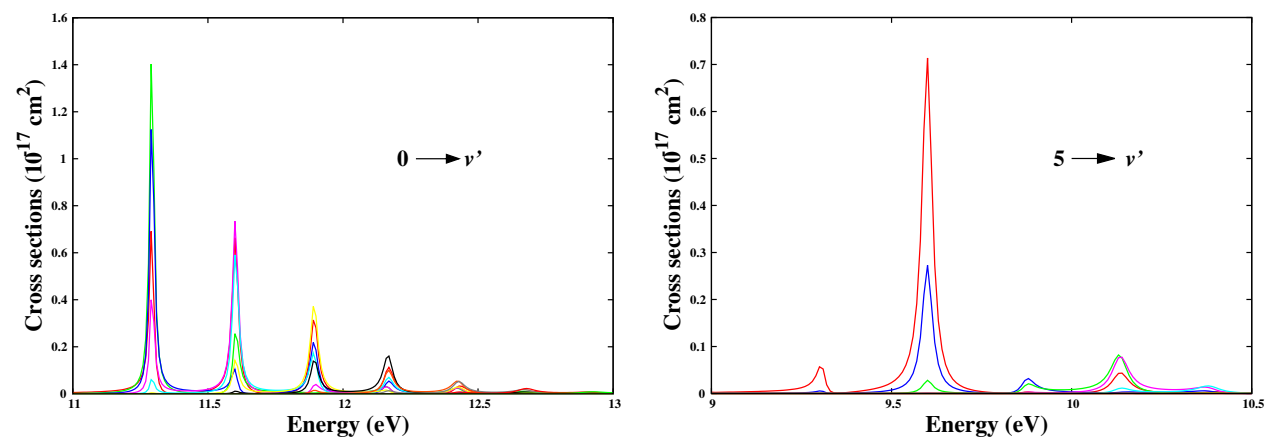

Figure 15. Electron- $\mathrm{H}_{2}$ RVE cross sections for the $v_{i}=0 \rightarrow v_{f}$ and $v_{i}=5 \rightarrow v_{f}$ transitions for $v_{f} \geq v_{i}$ [25].

according to the process

$$
\mathrm{H}_{2}\left(\mathrm{X}^{1} \Sigma_{\mathrm{g}}^{+}, v_{i}\right)+e \rightarrow \mathrm{H}_{2}^{-}\left({ }^{2} \Sigma_{\mathrm{g}}^{+}\right)^{*} \rightarrow \mathrm{H}_{2}\left(\mathrm{X}^{1} \Sigma_{\mathrm{g}}^{+}, v_{f}\right)+e .
$$

Potential curves for the Rydberg state and the corresponding widths were taken from Ref. [26], opportunely extrapolated to the asymptotic regions [27]. An example of the calculated cross sections is shown in Fig. 15.

The most evident feature of the two plots is the appearance of very sharp peaks which indicate the quasi-stable nature of the $\mathrm{H}_{2}^{-}\left({ }^{2} \Sigma_{\mathrm{g}}^{+}\right)$resonant electronic state, while the peak positions occur at the corresponding energy of vibrational levels. The calculations were extended to the cross sections for all the transitions with $v_{i} \leq v_{f} \leq 15$. Those for the reverse processes $\left(v_{i}>v_{f}\right)$ can be obtained by the principle of detailed balance [19].

All the cross section data for the processes illustrated in this contribution, along with the corresponding Maxwellian rate coefficients, not discussed here for space reason, can be accessed at the website http://users.ba.cnr.it/imip/cscpal38/phys4entry/database.html.

This work was performed under the auspices of the European Union Phys4Entry project funded under grant FP7SPACE-2009-1 242311. 


\section{References}

[1] M. Capitelli, I. Armenise, E. Bisceglie, D. Bruno, R. Celiberto, G. Colonna, G. D’Ammando, O. De Pascale, F. Esposito, C. Gorse, V. Laporta, A. Laricchiuta, Plasma Chem. Plasma Process. 32, 427 (2012)

[2] M. Capitelli, R. Celiberto, F. Esposito, A. Laricchiuta, Plasma Process. Polym. 6, 279 (2009)

[3] A. Bultel, B. G. Chéron, A. Bourdon, O. Motapon, and I. F. Schneider, Phys. Plasmas 13, 043502 (2006)

[4] M. Capitelli, R. Celiberto, G. Colonna, G. D’Ammando, O. De Pascale, P. Diomede, F. Esposito, C. Gorse, A. Laricchiuta, S. Longo and L. D. Pietanza, J. Phys. B: At. Mol. Opt. Phys. 43, 144025 (2010)

[5] V. Laporta, R. Celiberto and J. M. Wadehra, Plasma Sources Sci. Technol. 21, 055018 (2012)

[6] M. Allan, J. Phys. B: At. Mol. Phys. 18, 4511 (1985)

[7] V. Laporta, R. Celiberto and J. Tennyson, Plasma Sources Sci. Technol., 22, 025001 (2013)

[8] C. J. Noble, K. Higgins, G. Wöste, P. Duddy, P. G. Burke, P. J. O. Teubner, A. G. Middleton and M. J. Brunger, Phys. Rev. Lett. 76, 3534 (1996)

[9] M. Allan, J. Phys. B: At. Mol. Opt. Phys. 28, 5163 (1995)

[10] M. Allan, J. Phys. B: At. Mol. Phys. 38, 603 (2005)

[11] C. S. Trevisan, K. Houfek, Z. Zhang, A. E. Orel, C. W. McCurdy, and T. N. Rescigno, Phys. Rev. A 71, $052714(2005)$

[12] H. J. Werner et al 2009 MOLPRO version 2009.1, a package of ab initio programs; see http://www.molpro.net/

[13] V. Laporta, C. M. Cassidy, J. Tennyson and R. Celiberto, Plasma Sources Sci. Technol. 21, 045005 (2012)

[14] L. A. Morgan, J. Phys. B: At. Mol. Opt. Phys. 24, 4649 (1991)

[15] M. Allan, Phys. Rev. A 81, 042706 (2010)

[16] T. N. Rescigno, W. A. Isaacs, A. E. Orel, H. D. Meyer, and C. W. McCurdy, Phys. Rev. A 65, 032716 (2002)

[17] I. Čadez, F. Gresteau, M. Tron, and R. I. Hall, J. Phys. B: At. Mol. Opt. Phys. 10, 3821 (1977)

[18] R. Celiberto, V. Laporta, A. Laricchiuta, J. Tennyson, and J. M. Wadehra, The Open Plasma Physics Journal 7, 33 (2014)

[19] R. Celiberto, R. K. Janev and D. Reiter, Plasma Phys. Control. Fusion 54, 035012 (2012)

[20] K. Chakrabarti and J. Tennyson, Eur. Phys. J. D 66 (Issue 31, Topical issue: Electron/Positron Collision) (2012)

[21] R. Celiberto, K. L. Baluja and R. K. Janev, Plasma Sources Sci. Technol. 22, 015008 (2013)

[22] F. B. C. Machado and F. R. Ornellas, J. Chem. Phys. 94, 7237 (1991)

[23] J. Pitarch-Ruiz, J. Sánchez-Marin, A. M. Velasco and I. Martin, J. Chem. Phys. 129, 054310 (2008)

[24] J. N. Bardsley and J. M. Wadehra, Phys. Rev. A 20, 1398 (1979)

[25] R. Celiberto, R. K. Janev, V. Laporta, J. Tennyson and J. M. Wadehra, Phys Rev. A 88, 062701 (2013)

[26] D. T. Stibbe and J. Tennyson, J. Phys. B: At. Mol. Phys. 31, 815 (1998)

[27] R. Celiberto, R. K. Janev, J. M. Wadehra and A. Laricchiuta, Phys. Rev. A 77, 012714 (2008) 